\title{
Kissing may be an important and neglected risk factor for oropharyngeal gonorrhoea: a cross- sectional study in men who have sex with men
}

\author{
Eric P F Chow (D) 1,2 Vincent J Cornelisse (D) , ${ }^{1,2}$ Deborah A Williamson, ${ }^{3}$ David Priest, ${ }^{2}$ \\ Jane S Hocking, ${ }^{4}$ Catriona S Bradshaw, ${ }^{1,2}$ Tim R H Read, ${ }^{1,2}$ Marcus Y Chen, ${ }^{1,2}$ \\ Benjamin P Howden, ${ }^{3}$ Christopher K Fairley ${ }^{1,2}$
}

\begin{abstract}
- Additional material is published online only. To view please visit the journal online (http://dx.doi.org/10.1136/ sextrans-2018-053896)

${ }^{1}$ Central Clinical School, Monash University, Melbourne, Victoria, Australia

${ }^{2}$ Melbourne Sexual Health Centre, Alfred Health, Carlton, Victoria, Australia

${ }^{3}$ Microbiological Diagnostic Unit Public Health Laboratory, Department of Microbiology and Immunology, The University of Melbourne at The Peter Doherty Institute for Infection and Immunity, Melbourne, Victoria, Australia

${ }^{4}$ Melbourne School of Population and Global Health, University of Melbourne, Carlton, Victoria, Australia
\end{abstract}

Correspondence to Associate Professor Eric P F Chow, Melbourne Sexual Health Centre, Alfred Health, Carlton, VIC 3053, Australia; eric.chow@ monash.edu

Received 2 November 2018 Revised 3 March 2019 Accepted 24 March 2019 Published Online First 9 May 2019
ABSTRACT

Objectives A mathematical model suggested that a significant proportion of oropharyngeal gonorrhoea cases are acquired via oropharynx-to-oropharynx transmission (ie, tongue-kissing), but to date, no empirical study has investigated this. This study aimed to examine the association between kissing and oropharyngeal gonorrhoea among gay and bisexual men who have sex with men (MSM).

Methods MSM attending a public sexual health centre in Melbourne, Australia, between March 2016 and February 2017 were invited to participate in a brief survey that collected data on their number of male partners in the last 3 months, in three distinct categories: kissing-only (ie, no sex including no oral and/or anal sex), sex-only (ie, any sex without kissing), and kissingwith-sex (ie, kissing with any sex). Univariable and multivariable logistic regression analyses were performed to examine associations between oropharyngea gonorrhoea positivity by nucleic acid amplification tests and the three distinct partner categories.

Results A total of 3677 men completed the survey and were tested for oropharyngeal gonorrhoea. Their median age was 30 (IQR 25-37) and 6.2\% ( $n=229)$ had oropharyngeal gonorrhoea. Men had a mean number of 4.3 kissing-only, 1.4 sex-only, and 5.0 kissing-withsex partners in the last 3 months. Kissing-only and kissing-with-sex were associated with oropharyngeal gonorrhoea, but sex-only was not. The adjusted odds for having oropharyngeal gonorrhoea were 1.46-fold (95\% $\mathrm{Cl} 1.04$ to 2.06 ) for men with $\geq 4$ kissing-only partners and 1.81 -fold ( $95 \% \mathrm{Cl} 1.17$ to 2.79 ) for men with $\geq 4$ kissing-with-sex partners.

Conclusions These data suggest that kissing may be associated with transmission of oropharyngeal gonorrhoea in MSM, irrespective of whether sex also occurs.

Check for updates

(C) Author(s) (or their employer(s)) 2019. No commercial re-use. See rights and permissions. Published by BMJ.

To cite: Chow EPF Cornelisse VJ, Williamson DA, et al. Sex Transm Infect 2019:95:516-521

The rise in gonorrhoea cases among gay and bisexual men who have sex with men (MSM) is of substantial public health concern worldwide. Antibiotic-resistant Neisseria gonorrhoeae isolates have increasingly been documented in many countries, ${ }^{1}$ and a recent genomic analysis demonstrated transmit between sexual partners, ${ }^{2}$ raising the concern that gonorrhoea will become increasingly difficult to treat. ${ }^{3}$ To combat this trend, better evidence-based public health strategies are needed to prevent the spread of gonorrhoea. Public health messaging typically focuses on the promotion of condom use because most gonorrhoea is considered to be transmitted via penile-anal sex among MSM. ${ }^{4}$ However, a mathematical model published in 2017 has suggested that the transmission from the urethra to the oropharynx or the anorectum represents only a relatively small proportion of these incident infections. ${ }^{5}$

A number of pieces of evidence suggest that transmission from the oropharynx may be more common than previously thought. $N$. gonorrhoeae can be cultured from saliva, ${ }^{6-8}$ suggesting that the exchange of saliva between individuals may potentially transmit gonorrhoea. This idea is supported by the observation that receptive oro-anal contact (ie, rimming) and saliva use for lubrication during anal sex $^{9}$ are risk factors for anorectal gonorrhoea. ${ }^{4} 10$ Tongue-kissing is the most common sexual practice among MSM $^{11}$ and involves the exchange of saliva between MSM. Several case reports in the 1970 s suggested kissing as a mode of transmission for oropharyngeal gonorrhoea and led to the proposal that gonorrhoea could be transmitted this way. ${ }^{72-14}$ It has been proposed that gonorrhoea can be transmitted through kissing, ${ }^{15-17}$ but kissing has always been neglected as a risk factor for gonorrhoea transmission. To date, only two Australian epidemiological studies have examined the association between kissing and oropharyngeal gonorrhoea, ${ }^{18} 19$ and in both studies kissing was only associated with oropharyngeal gonorrhoea in unadjusted univariate analyses. However, both studies only examined kissing during sex, and neither study examined whether kissing occurred without sex. Accordingly, this study aimed to examine whether tongue-kissing (henceforth referred to as kissing) either alone or associated with sex is a risk factor for oropharyngeal gonorrhoea among MSM. that antibiotic-resistant strains of $N$. gonorrhoeae 
MSHC is the major public sexual health service in Victoria, Australia, which provides a free walk-in service to all individuals who attend. On arrival, all new patients and returning patients are asked to complete a questionnaire using a computer-assisted self-interview (CASI), which collects information on demographics and sexual practices.

Between March 2016 and February 2017, a brief survey, namely the 'Kissing' survey, was added after the routine clinical care questionnaire via CASI. All MSM who were asked to complete CASI were also invited to participate in the 'Kissing' survey. The 'Kissing' survey took less than 2 min to complete.

The 'Kissing' survey was designed to collect data on the participants' number of male partners in three distinct categories in relation to kissing in the last 3 months: (1) kissing-only partners (the number of men with whom the participant had only tongue-kissed without any other sexual contacts); (2) sex-only partners (the number of men with whom the participant had any sexual contact, but without tongue-kissing); and (3) kissing-with-sex partners (the number of men with whom the participant had tongue-kissed and had other sexual contact). For the purpose of this survey, sex was defined as oral or anal sex. Participants who agreed to participate in this survey were asked to provide consent by clicking 'yes' via CASI. A 'decline' option was also provided for men who did not want to participate. Ethics approval was obtained from the Alfred Hospital Ethics Committee, Melbourne, Australia (project number 69/16).

\section{Measurement}

For the present study, MSM were defined as men who have had any sexual contact with another man in the last 12 months. Transgender individuals were not eligible to participate in this survey. Demographic characteristics (eg, age and country of birth), known gonorrhoea contact, HIV status, and laboratory diagnosis of gonorrhoea were collected as part of routine clinical care. Oropharyngeal and anal swabs were taken with the swabs provided by the manufacturer. First pass urine samples were also collected. All specimens were tested by nucleic acid amplification test (NAAT) for N. gonorrhoeae using Aptima Combo $2 \AA$ Assay (Hologic Panther system; Hologic, San Diego, CA, USA).

\section{Statistical analysis}

Participants were included in the final analysis if they were male, aged $\geq 16$ years, had completed the 'Kissing' survey (ie, reported either having kissed or had sex with another man in the last 3 months), and had been tested for oropharyngeal gonorrhoea on the day when they completed the survey. Participants could participate in the study more than once during the study period. Given that the 'Kissing' survey collected data on the number of partners in the last 3 months, if the participant provided more than one response within 3 months, only the first response was included in the analysis.

Summary statistics of the study variables were calculated and reported. The mean and median of the three distinct categories of partners, stratified by age, were calculated. Negative binomial regression models were used to evaluate the differences between the mean number of partners among age groups. The Jonckheere-Terpstra test was used to evaluate the median number of partners among age groups. For the purpose of the present study, the number of partners was categorised into three groups: low (0-1 partner); medium ( $2-3$ partners); and high ( $\geq 4$ partners). We defined the medium group as 2-3 partners because in our study the median number of partners for kissing-only was 2 , and kissing-with-sex was 3 . Univariable and multivariable logistic

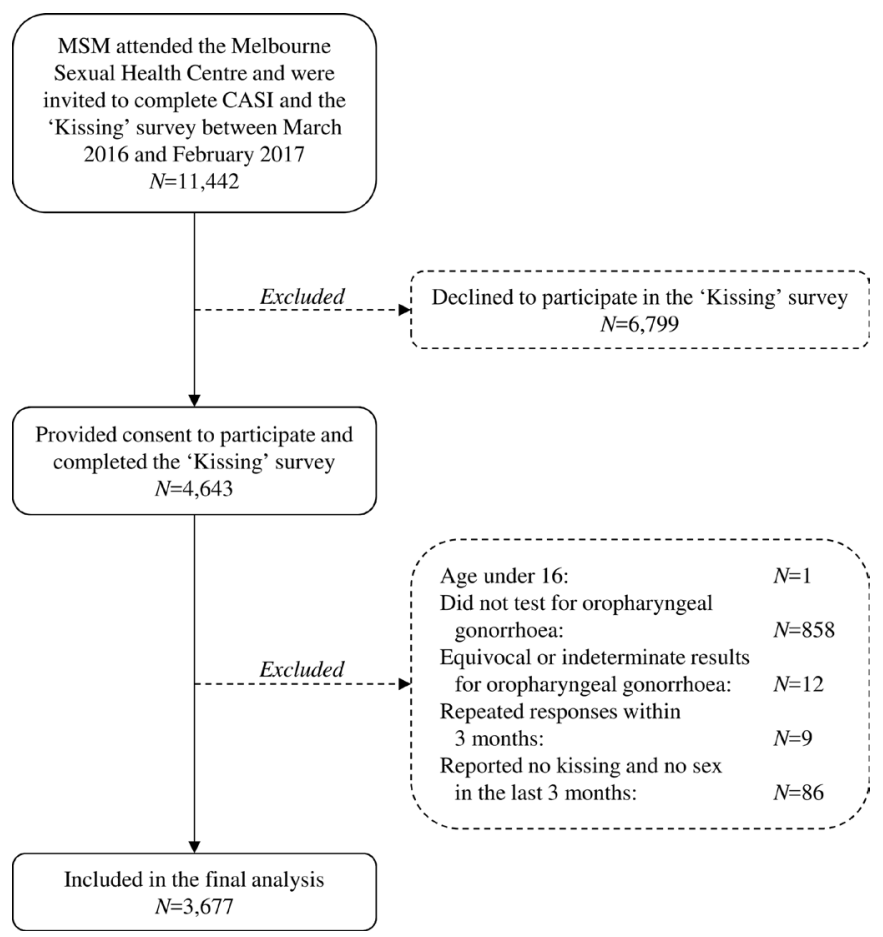

Figure 1 Flow chart illustrating the selection process for the final analysis. CASI, computer-assisted self-interview; MSM, men who have sex with men.

regression analyses were performed to examine associations between oropharyngeal gonorrhoea and the three distinct categories of partners, with generalised estimating equations used to address repeated-measures within individuals. Participants' characteristics with $p<0.10$ in the univariable analyses were considered as potential confounding factors and were included in the multivariable logistic regression. Crude and adjusted odds ratios $(\mathrm{OR})$ and the $95 \%$ confidence intervals $(95 \% \mathrm{CI})$ were calculated. All statistical analyses were conducted using Stata (version 14, College Station, Texas, USA).

\section{RESULTS}

In total, 11442 MSM were invited to complete the CASI during the study period and were automatically invited to participate in the 'Kissing' survey: 4643 men (40.6\%) completed the survey, 6799 men (59.4\%) declined to participate, and 966 responses did not meet the eligibility criteria and were excluded (figure 1). Thus, a total of 3677 survey responses from 3091 individual MSM were included in the final analysis. There was no significant difference in age between participants and non-participants $(\mathrm{p}=0.085)$.

Among the 3677 included responses, the median age was 30 (IQR 25-37), 57.1\% $(\mathrm{n}=2098)$ were Australian-born, and 2.5\% $(\mathrm{n}=92)$ had previously been diagnosed with HIV. The positivity for oropharyngeal gonorrhoea was 6.2\% (95\% CI 5.5\% to $7.1 \%$; 229/3677), anorectal gonorrhoea was $5.9 \%$ (95\% CI $5.2 \%$ to $6.8 \% ; 201 / 3389$ ), and urethral gonorrhoea was $2.7 \%$ (95\% CI $2.2 \%$ to $3.3 \%$; $98 / 3641)$. Almost all men $(95.2 \%$, $95 \%$ CI $94.5 \%$ to $95.9 \%$; $\mathrm{n}=3501$ ) had kissing-with-sex partners, and most men $(70.4 \%, 95 \% \mathrm{CI} 68.9 \%$ to $71.8 \%$; $\mathrm{n}=2587)$ had kissing-only partners, but less than half $(38.3 \%, 95 \%$ CI $36.7 \%$ to $39.9 \% ; \mathrm{n}=1407$ ) had sex-only partners in the last 3 months. There were 981 men $(26.7 \%$, 95\% CI $25.3 \%$ to $28.1 \%)$ who had all three categories of partners. A very small proportion of 
Table 1 Mean and median number of partners for kissing-only, sexonly and kissing-with-sex partners among 3677 men who have sex with men, stratified by age

\begin{tabular}{lllll}
\hline & $\begin{array}{l}\text { Mean (SD) } \\
\text { number of } \\
\text { partners }\end{array}$ & $\mathbf{P}_{\text {trend }}{ }^{*}$ & $\begin{array}{l}\text { Median (IQR) } \\
\text { number of } \\
\text { partners }\end{array}$ & $\mathbf{P}_{\text {trend }}{ }^{\dagger}$ \\
\hline Kissing-only partner & & & & \\
\hline $16-24$ & $5.07(7.85)$ & $<0.001$ & $3(1-6)$ & $<0.001$ \\
$25-34$ & $4.68(9.28)$ & & $2(0-5)$ & \\
$35-44$ & $3.97(9.49)$ & & $2(0-5)$ & \\
$\geq 45$ & $2.41(5.00)$ & & $0(0-3)$ & \\
All & $4.33(8.59)$ & - & $2(0-5)$ & - \\
Sex-only partner & & & & \\
$16-24$ & $0.79(2.04)$ & $<0.001$ & $0(0-1)$ & $<0.001$ \\
$25-34$ & $1.26(3.17)$ & & $0(0-1)$ & \\
$35-44$ & $1.69(4.13)$ & & $0(0-2)$ & \\
$\geq 45$ & $2.65(8.07)$ & & $1(0-3)$ & \\
All & $1.42(4.22)$ & - & $0(0-1)$ & \\
Kissing-with-sex partner & & & & \\
$16-24$ & $4.25(5.76)$ & 0.025 & $3(2-5)$ & \\
$25-34$ & $5.27(9.97)$ & & $3(2-5)$ & \\
$35-44$ & $5.64(10.43)$ & & $3(2-6)$ & \\
$\geq 45$ & $4.74(6.28)$ & & $3(1-5)$ & \\
All & $5.04(8.87)$ & - & $3(2-5)$ & - \\
\hline
\end{tabular}

${ }^{*} \mathrm{P}$ for trend was calculated from negative binomial regression model. tP for trend was calculated from Jonckheere-Terpstra test. $I Q R$, interquartile range; $S D$, standard deviation.

men in the last 3 months reported having only kissing-only partners $(1.4 \%, 95 \%$ CI $1.1 \%$ to $1.9 \% ; n=52)$ or sex-only partners $(2.6 \%, 95 \%$ CI $2.1 \%$ to $3.1 \% ; n=95)$.

Of the $52(1.4 \%)$ men who only had kissing-only partners, the positivity for gonorrhoea at the oropharynx was 3.8\% (95\% CI $0.5 \%$ to $13.2 \% ; 2 / 52)$, at the anorectum it was $2.3 \%(95 \% \mathrm{CI}$ $0.1 \%$ to $12.0 \% ; 1 / 44)$, and at the urethra it was $0 \%(95 \%$ CI $0 \%$ to $6.8 \% ; 0 / 52)$. Of the $95(2.6 \%)$ men who only had sex-only partners, the positivity for gonorrhoea at the oropharynx was $3.2 \%(95 \%$ CI $0.7 \%$ to $9.0 \% ; 3 / 95)$, at the anorectum it was $4.0 \%(95 \%$ CI $0.8 \%$ to $11.2 \% ; 3 / 75)$, and at the urethra it was $4.3 \%(95 \%$ CI $1.2 \%$ to $10.6 \% ; 4 / 93)$. The positivity of oropharyngeal gonorrhoea among the 95 men who only had sex-only partners was significantly lower compared with the total study population ( $3 \%$ vs $6 \% ; \mathrm{p}=0.020)$.

Among all men, the mean (SD) number of kissing-only partners was 4.3 (8.6), which was similar to the mean number of kissing-with-sex partners $(5.0(8.9))$. However, the mean number of sex-only partners was low (1.4 (4.2)). The mean and median number of kissing-only partners decreased significantly with increasing age $\left(\mathrm{p}_{\text {trend }}<0.001\right)$ (table 1$)$. In contrast, the mean and median number of sex-only partners increased significantly with increasing age $\left(\mathrm{p}_{\text {trend }}<0.001\right)$. The mean number of kissing-with-sex partners varied across different age groups but the median number did not change with age. The mean and median of all three categories of partners did not differ by HIV status. Supplementary figure S1 shows that the number of kissing-only partners was correlated with sex-only partners $(\rho=0.048$; $p=0.003)$ and kissing-with-sex partners $(\rho=0.392 ; p<0.001)$. A similar correlation between the number of sex-only partners and kissing-with-sex partners $(\rho=0.066 ; p<0.001)$ was also observed.

Having a greater number of kissing-only partners or a greater number of kissing-with-sex partners were significantly associated with oropharyngeal gonorrhoea in both univariable and multivariable analyses (table 2). After adjusting for potential confounding factors, the odds of having oropharyngeal gonorrhoea was 1.46 -fold (95\% CI 1.04 to 2.06 ) for men with $\geq 4$ kissing-only partners and 1.81 -fold (95\% CI 1.17 to 2.79 ) for men with $\geq 4$ kissing-with-sex partners, compared with men who had 0-1 partners in these respective categories. In contrast, the number of sex-only partners was not a significant risk factor for oropharyngeal gonorrhoea in either univariable or multivariable analyses.

The univariable analysis showed younger men had greater odds of having oropharyngeal gonorrhoea than older men, but this association disappeared in the multivariable analysis after adjusting for other confounding factors. In addition, the univariable analysis showed the odds of having oropharyngeal gonorrhoea were 2.32-fold (95\% CI 1.25 to 4.32) higher among HIV-positive men compared with HIV-negative men, but this association did not persist in the multivariable analysis after adjusting for potential confounding factors. Oropharyngeal gonorrhoea was not associated with the country of birth or sexual orientation.

\section{DISCUSSION}

This cross-sectional study provides the first empiric evidence that gonorrhoea may be transmitted from one man's oropharynx to another man's oropharynx through tongue-kissing. Previously, it has generally been assumed that oropharyngeal gonorrhoea can only be acquired from an infected penis or anus, not from another man's oropharynx ${ }^{16}$; hence, the importance of the oropharynx has been neglected in gonorrhoea transmission. Our results suggest kissing with or without sex may be a risk factor for oropharyngeal gonorrhoea. Our data align with the new proposed model that the oropharynx may play an important role in gonorrhoea transmission among MSM. ${ }^{15} 16$ Previous studies have shown that oropharyngeal gonorrhoea is more common in younger men, ${ }^{20-22}$ and we found that younger men kiss more sexual partners than older men. One implication of this finding is that potential interventions such as antiseptic mouthwash, if shown to be effective against oropharyngeal gonorrhoea, could provide a non-condom and non-antibiotic-based intervention for gonorrhoea control. This is particularly relevant in the context of recent reports of highly-resistant N. gonorrhoeae, ${ }^{23}$ and known challenges associated with the antimicrobial treatment of oropharyngeal gonorrhoea. ${ }^{2425}$

This study has some limitations that we wish to address. Firstly, selection bias might have occurred, given this study was conducted at a single urban sexual health centre in Melbourne, which may not represent the entire MSM population in Australia and in other settings. Secondly, about $60 \%$ of MSM attending our centre declined to participate in the 'Kissing' survey via CASI. It is possible that sexual practices differed between those men who participated in the study and those who declined. However, the oropharyngeal gonorrhoea positivity in this study $(6.2 \%)$ is similar to our clinic population $(7.0 \%$ among all MSM attending MSHC during the study period; $p=0.104)$, suggesting that our participants comprised a representative sample from the clinic population. Thirdly, we did not measure the number of penile-oral sex or anal sex partners but only included these indirectly as part of the partners reporting 'any sex'. The definition of any sex was the same in all of the three key comparison groups we used in our survey. The strong relationships between kissing-only and kissing-with-sex partners, and between kissing-only and sex-only partners, suggest that the evaluated odds of having 
Table 2 Risk factors associated with oropharyngeal gonorrhoea positivity among 3677 men who have sex with men

\begin{tabular}{|c|c|c|c|c|c|c|}
\hline Risk factors & Number of individuals & $\begin{array}{l}\text { Oropharyngeal } \\
\text { gonorrhoea positive } \\
\text { n (\%) }\end{array}$ & Crude OR $(95 \% \mathrm{CI})$ & $P$ value & Adjusted OR $(95 \% \mathrm{Cl})$ & $P$ value \\
\hline \multicolumn{4}{|c|}{ Number of kissing-only partners in the last 3 months } & $<0.001^{*}$ & & $0.029^{*}$ \\
\hline $0-1$ & 1634 & $73(4.5 \%)$ & 1 (Ref) & - & 1 (Ref) & - \\
\hline $2-3$ & 747 & $44(5.9 \%)$ & 1.34 (0.91 to 1.97$)$ & - & 1.30 (0.87 to 1.95$)$ & - \\
\hline$\geq 4$ & 1296 & $112(8.6 \%)$ & 2.02 (1.49 to 2.74$)$ & - & 1.46 (1.04 to 2.06$)$ & - \\
\hline \multicolumn{4}{|c|}{ Number of sex-only partners in the last 3 months } & $0.068^{*}$ & & $0.541^{*}$ \\
\hline $0-1$ & 2776 & $159(5.7 \%)$ & 1 (Ref) & - & 1 (Ref) & - \\
\hline $2-3$ & 506 & $38(7.5 \%)$ & $1.33(0.92$ to 1.92$)$ & - & $1.39(0.95$ to 2.02$)$ & - \\
\hline$\geq 4$ & 395 & $32(8.1 \%)$ & 1.44 (0.97 to 2.14$)$ & - & 1.14 (0.75 to 1.73$)$ & - \\
\hline \multicolumn{4}{|c|}{ Number of kissing-with-sex partners in the last 3 months } & $<0.001^{*}$ & & $0.008^{*}$ \\
\hline $0-1$ & 849 & $32(3.8 \%)$ & 1 (Ref) & - & 1 (Ref) & - \\
\hline $2-3$ & 1243 & $63(5.1 \%)$ & $1.36(0.88$ to 2.10$)$ & - & 1.14 (0.72 to 1.79$)$ & - \\
\hline$\geq 4$ & 1585 & $134(8.5 \%)$ & 2.36 (1.59 to 3.50$)$ & - & 1.81 (1.17 to 2.79$)$ & - \\
\hline \multicolumn{4}{|l|}{ Age (years) } & $0.041^{*}$ & & $0.076^{*}$ \\
\hline $16-24$ & 805 & $56(7.0 \%)$ & 1.63 (1.99 to 2.70$)$ & - & 1.55 (0.91 to 2.61$)$ & - \\
\hline $25-34$ & 1706 & $113(6.6 \%)$ & 1.55 (0.97 to 2.46$)$ & - & $1.47(0.91$ to 2.39$)$ & - \\
\hline $35-44$ & 667 & $38(5.7 \%)$ & 1.32 (0.77 to 2.26$)$ & - & 1.25 (0.72 to 2.16$)$ & - \\
\hline$\geq 45$ & 499 & $22(4.4 \%)$ & 1 (Ref) & - & 1 & - \\
\hline \multicolumn{7}{|l|}{ HIV status } \\
\hline Negative & 3585 & $217(6.1 \%)$ & 1 (Ref) & - & 1 (Ref) & - \\
\hline Positive & 92 & $12(13.0 \%)$ & 2.32 (1.25 to 4.32$)$ & 0.008 & 1.86 (0.96 to 3.58$)$ & 0.064 \\
\hline \multicolumn{7}{|c|}{ Known gonorrhoea contact } \\
\hline No & 3536 & $196(5.5 \%)$ & 1 (Ref) & - & 1 (Ref) & - \\
\hline Yes & 141 & $33(23.4 \%)$ & 5.20 (3.43 to 7.89$)$ & $<0.001$ & 4.67 (3.05 to 7.16$)$ & $<0.001$ \\
\hline \multicolumn{7}{|c|}{ Country of birth } \\
\hline Australia & 2098 & $131(6.2 \%)$ & 1 (Ref) & - & - & - \\
\hline Overseas & 1409 & $89(6.3 \%)$ & 1.01 (0.77 to 1.33 ) & 0.932 & - & - \\
\hline Unknown & 170 & $9(5.3 \%)$ & 0.84 (0.42 to 1.67$)$ & 0.612 & - & - \\
\hline \multicolumn{7}{|c|}{ Sexual orientationt } \\
\hline Bisexual & 342 & $17(5.0 \%)$ & 1 (Ref) & - & - & - \\
\hline Gay & 3335 & $212(6.4 \%)$ & 1.29 (0.78 to 2.14$)$ & 0.325 & - & - \\
\hline
\end{tabular}

${ }^{*} \mathrm{P}$ for trend for categorical variables.

tSexual orientation was categorised as gay (only had sex with men) or bisexual (had sex with both men and women). This categorisation was not based on self-identity. OR, odds ratio.

oropharyngeal gonorrhoea among men with a higher number of kissing partners could have been caused by other sexual behaviours that are associated with oropharyngeal gonorrhoea, namely fellatio. Fourthly, gonorrhoea was screened by NAAT. NAAT has a higher sensitivity but lower specificity than culture; therefore, we were not able to identify whether the organism was viable or not. Finally, we did not collect other data on the characteristics of kissing behaviours such as the duration, the number of female partners kissed by bisexual men, or the number of acts of oral sex, all of which could influence the risk of transmission.

This study has several strengths that make it stand apart from the previous two epidemiological studies that examined associations between kissing and oropharyngeal gonorrhoea. ${ }^{18} 19$ First, this is the first gonorrhoea study to ask about kissing-only partners. The two previous studies only asked about the number of kissing partners among sexual partners and did not ask about 'kissing-only' partners. We found that MSM have similar numbers of kissing-only and kissing-with-sex partners. Hence, the previous two studies did not include half of the exposure to kissing, which is likely to be why they did not find an independent association between kissing and oropharyngeal gonorrhoea. Second, this is the largest study on kissing and oropharyngeal gonorrhoea to date. We included more than 3000 participants, compared with previous studies conducted by Cornelisse et al $(\mathrm{N}=531),{ }^{18}$ and Templeton et al $(\mathrm{N}=1427) .{ }^{19}$ Thirdly, we asked the participants to report the number of partners within a 3 month period. This timeframe is consistent with the study conducted by Cornelisse et $a l{ }^{18}$ but Templeton's study used a 6 month period. ${ }^{19}$ We believe it is more accurate to collect the number of partners in a 3 month period because previous studies show that the duration of untreated oropharyngeal gonorrhoea is unlikely to be more than 3 months. ${ }^{6222627}$ A systematic review published in 2016 estimated the duration of oropharyngeal gonorrhoea to be about 3-4 months. ${ }^{27}$ Moreover, Priest et al have shown that oropharyngeal gonorrhoea is only associated with partners in the last 3 months and not partners between 4 and 12 months ago. ${ }^{22}$

If kissing were the leading risk factor for oropharyngeal gonorrhoea, it might be expected that men who have sex-only partners would have a lower prevalence of oropharyngeal gonorrhoea because only a small proportion of incident oropharyngeal gonorrhoea cases are acquired from the urethra or anus. ${ }^{5}$ In our study, the oropharyngeal gonorrhoea positivity among the 95 men who reported having sex-only partners and no male kissing partners was significantly lower compared with the total study population $(3 \%$ vs $6 \% ; \mathrm{p}=0.020)$. Furthermore, it 
is reasonably hypothesised that men who only had kissing-only partners would only have gonorrhoea in the oropharynx but not at the anorectum and urethra. Of the 52 men who only had kissing-only partners, two men had oropharyngeal gonorrhoea, one man had anorectal gonorrhoea and none of them had urethral gonorrhoea. We performed a chart review on the man who had anorectal gonorrhoea and he had reported that he had anal sex in the last 12 months but not in the last 3 months; this is consistent with previous studies showing that the duration of anorectal gonococcal infection could last for 12 months. ${ }^{22}{ }^{27}$ We would advocate caution in drawing too many conclusions from these findings because they come from very small subsets (1.4$2.6 \%)$ of individuals in the study. However, it is suggested that not all oropharyngeal gonorrhoea is due to kissing and that some of it may be due to oro-anal or oro-penile sexual contacts. ${ }^{19} 28$ Future longitudinal studies would be required to infer causation between kissing and oropharyngeal gonorrhoea.

Consistent with previous research, ${ }^{20-22}$ oropharyngeal gonorrhoea positivity displays a strong age pattern, with younger men being at significantly greater risk. Previous studies have hypothesised that this age pattern in oropharyngeal gonorrhoea positivity could be due to the age pattern in kissing ${ }^{18}$ but no previous study has confirmed this correlation. In our study, this age pattern was present in the univariable analysis $\left(\mathrm{p}_{\text {trend }}=0.041\right)$ but not in the multivariable analysis $\left(\mathrm{p}_{\text {trend }}=0.076\right)$ after adjusting for potential confounding factors including kissing. We found that younger men have more kissing-only partners than older men, which is consistent with a previous study in the USA showing younger men are more likely to kiss their partners than older men. ${ }^{11}$ More research is required to further understand why younger men are at an increased risk of oropharyngeal gonorrhoea.

It is clearly challenging to promote a public health message advocating MSM do not kiss to improve gonorrhoea prevention and control. Not only is kissing common (in a US survey $75 \%$ of MSM had kissed in the last 12 months), but it is the single most common sexual practice among MSM. ${ }^{11}$ A previous Australian qualitative study showed that most MSM consider kissing to be very important for intimacy, ${ }^{29}$ and only a small proportion (11\%) would consider not kissing if this reduced their risk of oropharyngeal gonorrhoea. ${ }^{30}$ However, there are other sexual practices that men would be willing to forgo (eg, oro-anal sex) which may lessen the risk of transmission from the oropharynx to the anus. In the absence of an effective gonorrhoea vaccine, an intervention targeting the oropharynx is required to reduce the burden of gonorrhoea. A small randomised controlled trial and an in vitro experiment have shown that a one-off use of antiseptic mouthwash could inhibit

\section{Key messages}

- Gay and bisexual men who have sex with men (MSM) had a similar number for kissing-only and kissing-with-sex partners in the last 3 months.

- Kissing with and without sex may be a significant risk factor for oropharyngeal gonorrhoea in MSM, whereas sex alone is not a risk factor for oropharyngeal gonorrhoea.

- The adjusted odds for having oropharyngeal gonorrhoea for MSM with $\geq 4$ kissing-only and $\geq 4$ kissing-with-sex partners were 1.46 -fold and 1.81 -fold, respectively.

- Our data provide the first empiric evidence that gonorrhoea may be transmitted from one man's oropharynx to another man's oropharynx through tongue-kissing. the growth of N. gonorrhoeae among MSM diagnosed with oropharyngeal gonorrhoea by culture. ${ }^{31}$ It is possible that the use of mouthwash could potentially reduce the bacterial load of N. gonorrhoeae in the oropharynx and saliva immediately, and thus reduce incident cases at a population level. A larger multicentre randomised controlled trial is now underway in Australia to examine whether daily use of mouthwash could prevent oropharyngeal gonorrhoea and the findings from this trial will be available in $2019 .^{32}$

This study adds to previous case reports and case series and suggests that kissing may be a significant risk factor for oropharyngeal gonorrhoea in MSM. Research into gonorrhoea transmission between oropharynges is currently being neglected. Our data provide additional evidence to support the newly proposed and as yet hypothetical model for gonorrhoea transmission that, if true, would open up preventive options such as antibacterial mouthwash that do not rely on condoms.

\section{Handling editor Khalil G Ghanem}

Twitter Follow Eric Chow @EricPFChow

Acknowledgements The authors would like to acknowledge Afrizal Afrizal for his assistance with data extraction and Jun Kit Sze for his assistance in implementing the 'Kissing' survey on the computer-assisted self-interviewing system at the Melbourne Sexual Health Centre.

Contributors CKF proposed the idea to examine the role of kissing in gonorrhoea transmission. EPFC and CKF conceived the study and designed the questions for the 'Kissing' survey. EPFC and DP conducted the statistical analysis. EPFC wrote the first draft of the manuscript. All authors assisted with data interpretation and critically revised it for important intellectual content and read and approved the final version of the manuscript.

Funding EPFC, DAW and TRHR are supported by the Early Career Fellowships from the Australian National Health and Medical Research Council (NHMRC) (1091226, 1123854 and 1091536 respectively) and NHMRC ProgramProgramme grant (568971). BPH is supported by an NHMRC Practitioner Fellowship (1105905). VIC is supported by a Research Training Scheme Scholarship from the Australian Government's Department of Education and Training.

Competing interests None declared.

Patient consent for publication Not required.

Ethics approval Ethical approval was obtained from the Alfred Hospital Ethics Committee, Melbourne, Australia (project number 69/16).

Provenance and peer review Not commissioned; externally peer reviewed.

\section{ORCID iDs}

Eric P F Chow http://orcid.org/0000-0003-1766-0657

Vincent J Cornelisse http://orcid.org/0000-0003-0657-9677

\section{REFERENCES}

1 Lewis DA. Global resistance of Neisseria gonorrhoeae: when theory becomes reality. Curr Opin Infect Dis 2014;27:62-7.

2 Kwong JC, Chow EPF, Stevens K, et al. Whole-genome sequencing reveals transmission of gonococcal antibiotic resistance among men who have sex with men: an observational study. Sex Transm Infect 2018;94:151-7.

3 Barbee LA. Preparing for an era of untreatable gonorrhea. Curr Opin Infect Dis 2014:27:282-7.

4 Jin F, Prestage GP, Mao L, et al. Incidence and risk factors for urethral and anal gonorrhoea and Chlamydia in a cohort of HIV-negative homosexual men: the Health in Men Study. Sex Transm Infect 2007:83:113-9.

5 Zhang L, Regan DG, Chow EPF, et al. Neisseria gonorrhoeae transmission among men who have sex with men: an anatomical site-specific mathematical model evaluating the potential preventive impact of mouthwash. Sex Transm Dis 2017;44:586-92.

6 Hutt DM, Judson FN. Epidemiology and treatment of oropharyngeal gonorrhea. Ann Intern Med 1986;104:655-8.

7 Hallqvist L, Lindgren S. Gonorrhoea of the throat at a venereological clinic. Incidence and results of treatment. Sex Transm Infect 1975;51:395-7.

8 Chow EPF, Tabrizi SN, Phillips S, et al. Neisseria gonorrhoeae bacterial DNA load in the pharynges and saliva of men who have sex with men. J Clin Microbiol 2016:54:2485-90.

9 Butler LM, Osmond DH, Jones AG, et al. Use of saliva as a lubricant in anal sexual practices among homosexual men. J Acquir Immune Defic Syndr 2009;50:162-7. 
10 Chow EPF, Cornelisse VJ, Read TRH, et al. Saliva use as a lubricant for anal sex is a risk factor for rectal gonorrhoea among men who have sex with men, a new public health message: a cross-sectional survey. Sex Transm Infect 2016;92:532-6.

11 Rosenberger JG, Reece M, Schick V, et al. Sexual behaviors and situational characteristics of most recent male-partnered sexual event among gay and bisexually identified men in the United States. J Sex Med 2011;8:3040-50.

12 Willmott FE. Transfer of gonococcal pharyngitis by kissing? Sex Transm Infect $1974 ; 50: 317-8$

13 Bro-Jorgensen A, Jensen T. Gonococcal pharyngeal infections. Report of 110 cases. Sex Transm Infect 1973:49:491-9.

14 Tice AW, Tice AW, Jr. Pharyngeal gonorrhea. JAMA 1981;246:2717-9.

15 Fairley CK, Hocking JS, Zhang L, et al. Frequent transmission of gonorrhea in men who have sex with men. Emerg Infect Dis 2017;23:102-4.

16 Fairley CK, Zhang L, Chow EPF. New thinking on gonorrhoea control in MSM: are antiseptic mouthwashes the answer? Curr Opin Infect Dis 2018;31:45-9.

17 Cornelisse VJ, Williamson D, Zhang L, et al. Evidence for a new paradigm of gonorrhoea transmission: cross-sectional analysis of Neisseria gonorrhoeae infections by anatomical site in both partners in 60 male couples. Sex Transm Infect 2019:95:437-42.

18 Cornelisse VJ, Walker S, Phillips T, et al. Risk factors for oropharyngeal gonorrhoea in men who have sex with men: an age-matched case-control study. Sex Transm Infect. 2018;94:359-64.

19 Templeton DJ, Jin F, McNally LP, et al. Prevalence, incidence and risk factors for pharyngeal gonorrhoea in a community-based HIV-negative cohort of homosexual men in Sydney, Australia. Sex Transm Infect 2010;86:90-6.

20 Chow EPF, Walker S, Read TRH, et al. Self-reported use of mouthwash and pharyngeal gonorrhoea detection by nucleic acid amplification test. Sex Transm Dis 2017:44:593-5.

21 Chow EPF, Tomnay J, Fehler G, et al. Substantial increases in Chlamydia and gonorrhea positivity unexplained by changes in individual-level sexual behaviors among men who have sex with men in an Australian sexual health service from 2007 to 2013. Sex Transm Dis 2015;42:81-7.

22 Priest $\mathrm{D}$, Read TRH, Chen MY, et al. Only recent sexual partners contribute to oropharyngeal gonorrhoea positivity: the number of sexual partners over different time periods as an indicator of gonorrhoea and Chlamydia infection duration among men who have sex with men. Sex Health 2018;15:342-9.

23 Lahra MM, Martin I, Demczuk W, et al. Cooperative recognition of internationally disseminated ceftriaxone-resistant Neisseria gonorrhoeae strain. Emerg Infect Dis 2018;24.

24 Poncin T, Fouere S, Braille A, et al. Multidrug-resistant Neisseria gonorrhoeae failing treatment with ceftriaxone and doxycycline in France, November 2017. Eurosurveillance 2018;23.

25 Chen MY, Stevens K, Tideman R, et al. Failure of $500 \mathrm{mg}$ of ceftriaxone to eradicate pharyngeal gonorrhoea, Australia. J Antimicrob Chemother 2013;68:1445-7.

26 Wallin J, Siegel MS. Pharyngeal Neisseria gonorrhoeae: coloniser or pathogen? BMJ 1979;1:1462-3.

27 Chow EPF, Camilleri S, Ward C, et al. Duration of gonorrhoea and Chlamydia infection at the pharynx and rectum among men who have sex with men: a systematic review. Sex Health 2016;13:199-204.

28 Barbee LA, Khosropour CM, Dombrowski JC, et al. An estimate of the proportion of symptomatic gonococcal, chlamydial and non-gonococcal non-chlamydial urethritis attributable to oral sex among men who have sex with men: a case-control study. Sex Transm Infect 2016;92:155-60.

29 Walker S, Bellhouse C, Fairley CK, et al. Pharyngeal gonorrhoea: the willingness of Australian men who have sex with men to change current sexual practices to reduce their risk of transmission-a qualitative study. Plos One 2016;11:e0164033.

30 Chow EPF, Walker S, Phillips T, et al. Willingness to change behaviours to reduce the risk of pharyngeal gonorrhoea transmission and acquisition in men who have sex with men: a cross-sectional survey. Sex Transm Infect 2017;93:499-502.

31 Chow EP, Howden BP, Walker S, et al. Antiseptic mouthwash against pharyngeal Neisseria gonorrhoeae: a randomised controlled trial and an in vitro study. Sex Transm Infect 2017;93:88-93.

32 Chow EPF, Walker S, Hocking JS, et al. A multicentre double-blind randomised controlled trial evaluating the efficacy of daily use of antibacterial mouthwash against oropharyngeal gonorrhoea among men who have sex with men: the OMEGA (Oral Mouthwash use to Eradicate GonorrhoeA) study protocol. BMC Infect Dis 2017; 17:456. 